\title{
Straight Skeleton Computation Optimized for Roof Model Generation
}

\author{
Kenichi Sugihara \\ Gifu Keizai University \\ 5-50 Kitagata-chou \\ Ogaki city, Gifu-Pref., 503-8550, Japan \\ sugihara@gifu-keizai.ac.jp
}

\begin{abstract}
3D building models with roofs are important in several fields, such as urban planning and BIM (Building Information Model). However, enormous time and labor are required to create these 3D models. In order to automate laborious steps, a GIS and CG integrated system is proposed for the automatic generation of 3D building models, based on building polygons (building footprints) on digital maps. The generation is implemented through straight skeleton computation, in which three events ('Edge' and 'Split', 'Vertex' events) were proposed. In the computation process, usually three edges propagate into a node. Often it causes an acute angle shape that is not appropriate for roof boards. To avoid the inappropriate shape, in this paper, methodologies are proposed for adding 'Line segment' events besides the conventional events, and monotone polygon nodes sorting.
\end{abstract}

\section{Keywords}

automatic generation, 3D building model, straight skeleton, building footprint, GIS.

\section{INTRODUCTION}

3D town models, such as the one shown in Fig.1 right, are important in urban planning and architectural design, e.g., BIM (Building Information Model). However, enormous time and labor are required to create these 3D models, using 3D modeling software such as $3 \mathrm{ds}$ Max or SketchUp. For example, when manually modeling a house with roofs by Constructive Solid Geometry (CSG), one must follow the following laborious steps:

(1) Generation of geometric primitives of proper size, such as boxes, prisms or polyhedra that will form parts of a house (2) Boolean operations are applied to these primitives to form the shapes of parts of a house such as making rectangular holes in a building body for doors and windows (3) Horizontal and vertical rotation of parts of a house (4) Placing the parts of a house to appropriate positions (5) Texture mapping onto these parts.

In order to save these laborious steps, a GIS and CG integrated system that automatically generates $3 \mathrm{D}$

\footnotetext{
Permission to make digital or hard copies of all or part of this work for personal or classroom use is granted without fee provided that copies are not made or distributed for profit or commercial advantage and that copies bear this notice and the full citation on the first page. To copy otherwise, or republish, to post on servers or to redistribute to lists, requires prior specific permission and/or a fee.
}

building models is proposed, based on building polygons (building footprints) on a digital map as shown in Fig.1 left and Fig.7a, which show most building polygons' edges meet at right angles (orthogonal polygon). An orthogonal polygon can be divided or separated into a set of rectangles. The proposed integrated system divides orthogonal building polygons into a set of rectangles and places rectangular roofs and box-shaped building bodies on these rectangles. In the digital map, however, building polygons are not always orthogonal. In either orthogonal or non-orthogonal polygons, the methodology is proposed for automatically creating general shaped roofs by the straight skeleton computation defined by Aichholzer et al. [Aic95].

In their proposal, two events ('Edge' and 'Split' events described in section 4) will occur during shrinking process. Besides two events, Eppstein et al. [Epp99] suggested a 'Vertex' event in which two or more reflex vertices reach the same point simultaneously. A reflex vertex is a vertex whose internal angle is greater than 180 degrees. However, some roofs are not created by these three events proposed. In our paper, the methodology was proposed for constructing roof models by assuming 'the Third event' in which a reflex vertex runs into the edge, but the other split polygon is collapsed into a node (an Edge event happens in the Split event at the same time).

In this paper, a methodology is proposed for adding a 'Line segment' event besides the conventional events. 


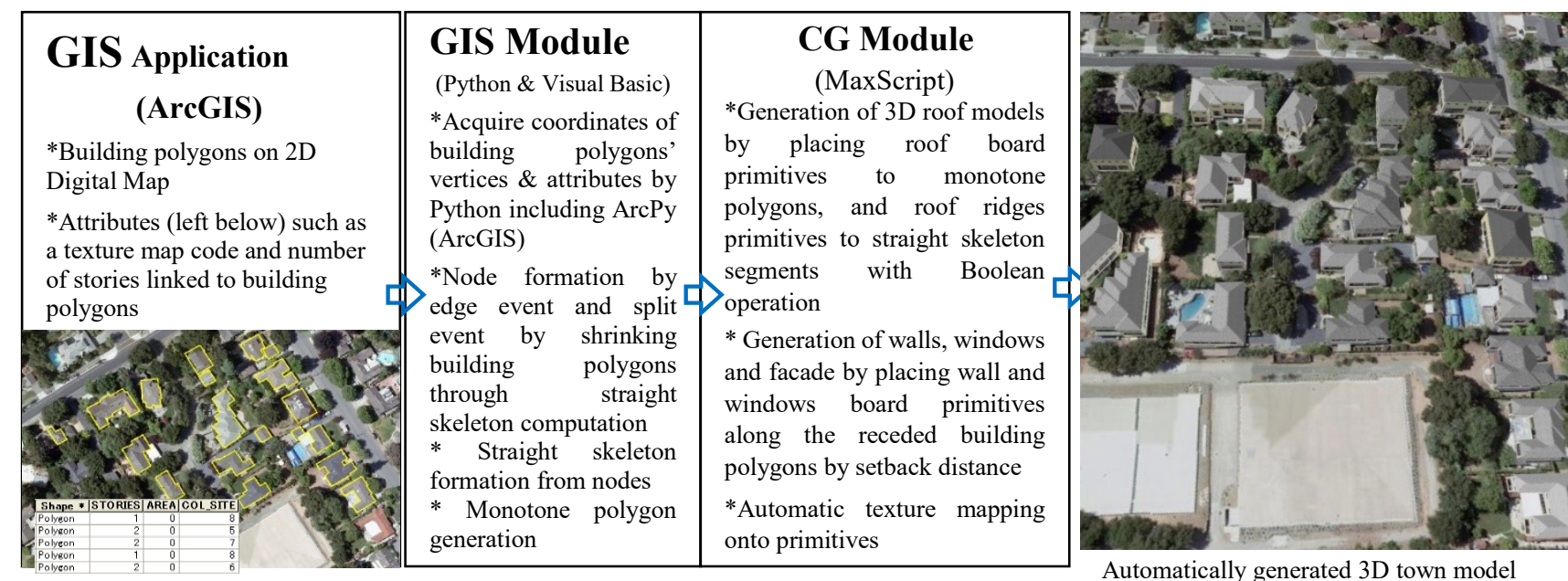

Figure 1: Pipeline of Automatic Generation for 3D Building Models by Straight Skeleton Computation

The shrinking process continues if polygons split have non-zero area. The shrinking process ends when polygons split fall into 'Vertex' or 'Line segment' since they have no area. Consequently, a 'Line segment' can be a resulting shape of shrinking procedure, and we will classify a 'Line segment' event in which two line segments collapse into one line segment.

Usually three edges propagate into a node. Often it causes an acute angle shape that is not appropriate for roof boards shown in Fig.3. To avoid the inappropriate shape, a 'Line segment' event will be proposed for straight skeleton computation. We also propose 'monotone polygon nodes sorting' by which not selfintersecting monotone polygons are formed, where 'monotone polygons' are the areas divided by a straight skeleton, as shown in Fig.2c.

\section{RELATED WORK}

Since 3D building models are used for several different purposes, such as urban planning, archaeological reconstruction and game industries, various types of technologies, ranging from computer vision, computer graphics, photogrammetry, and remote sensing, have been proposed and developed for creating 3D building models. When 3D building models are used for urban planning or reconstruction of ancient cities, we will make non-existent building models which we cannot take a picture, and therefore these models are not created by CV, photogrammetry, and remote sensing. So, we focus on procedural modeling, especially roof creation by straight skeleton computation. Procedural modeling is an effective technique to create $3 \mathrm{D}$ models from sets of rules such as L-systems, fractals, and generative modeling language [Par01]. Mueller et al. [Mue06] have created an archaeological site of Pompeii and a suburbia model of Beverly Hills by using a shape grammar. They import building footprints from a GIS database and try to classify imported polygons as basic shapes in their shape vocabulary. In case of misclassification, they use a general extruded footprint together with a general roof obtained by the straight skeleton algorithm defined by a continuous shrinking process [Aic95].

The straight skeleton algorithm is useful in generating hipped roofs, since its resulting 'monotone polygons' correspond to the roof boards of a hipped roof. The downside is that the generated roof is only one of many possible roofs, and there is no way to get another roof [Ede14]. To overcome the downside, a new generalization of straight skeletons is proposed by Helda et al. [Hel17], introducing additively-weighted straight skeletons. An additively-weighted straight skeleton is the result of a wavefront-propagation process where wavefront edges do not necessarily start to move at the begin of the propagation, resulting in an automated generation of roofs in which the individual facets have different inclinations and start at different heights.

By using the straight skeleton, Kelly et al. [Kel11] present a user interface for the exterior of architectural models to interactively specify procedural extrusions. They use a profile editor which controls the sweeping of a plane from the base of the building footprint, and they finally construct a wide variety of roofs and a complex architecture.

By these interactive modeling, 3D building models with plausible detailed façade can be achieved. However, the limitation of these modeling is the large amount of user interaction involved [Jia09], and the models created are 'surface models' by sweeping or extruding dependent on edited profiles, or revolving $2 \mathrm{D}$ primitive geometries. However, when creating 3D building models for architectural design and BIM, 3D building models should be made up of solid geometries primitives which will be parts of the building, created through Boolean operation. Thus, the 
GIS and CG integrated system that automatically generates 3D building models immediately by CSG (Constructive Solid Geometry) is proposed. Although surface models can have 'beautifully curved roof' by interactive procedural modeling, in reality these roofs are consisted of hundreds of narrow flat boards in most building design. These narrow boards will be properly placed along the roof curve.

\section{PIPELINE of AUTOMATIC GENERATION}

As the pipeline of automatic generation is shown in Fig.1, the source of 3D models is a digital map that contains building polygons linked with attributes data, such as the number of stories and the type of roof, shown in Fig.1 left below. The maps are then preprocessed at the GIS module, and the CG module finally generates the 3D building model.

The preprocessing at the GIS module includes the procedures as follows: (1) Calculate the minimum receding distance for an Edge event (including a Third and Line segment event). Until the Edge event occur, check if Split event happens by starting continuous shrinking process. (2) Start continuous shrinking process in which edges of the polygon move inward, parallel to themselves at a constant speed (Fig.2a\&2b). (3) Detect any event such as a Split, Edge or Line segment event during shrinking process, and formation of nodes by these events. The position of the node is calculated by the intersection of angular bisectors. (4) Inherit and store three or more original edges' ID (e.g. edgN in Fig.2a) linked to the node during the shrinking process in which the topology of the polygon will change. In shrinking process, Fig.2b shows edg2 firstly disappears into Node1, and two edges (edg8 \& 9) secondly result in Node2. Since at least three original edges sweep into the node, edg1,2 \& 3 propagation result in Node1, and edg4,5\& 10 propagation result in Node3 (by Split event). (5) Every (original) edge will inquire 'each node' having three or more ID to find out which node has the same original edge ID. If so, then nodes of the same ID are collected and the set of nodes are sorted according to the edge vector to form 'monotone polygon' and the straight skeleton. (6) Calculate the length, width, center position and inclination of the bounding rectangle for 'monotone polygon'. (7) Export the coordinates of polygons' vertices, 'monotone polygons' information, and attributes of buildings.

In these procedures, the areas divided by a straight skeleton are called as 'monotone polygons' shown in Fig.2c, and to get 'monotone polygons', the set of the nodes belonging to the same original edge will be aligned depending on the coordinate value on the axis parallel to each original edge vector (the 'node vector projections' onto the original edge vector). These nodes are coplanar and will form roof boards for a 3D building model.

As shown in Fig.1, the CG module receives the preprocessed data that the GIS module exports, generating 3D building models. In GIS module, the system measures the length and inclination of the bounding rectangle for the monotone polygon that will be a roof board. The CG module generates a bounding box of the length and width, measured in GIS module. The monotone polygons will be converted into primitives, i.e., thin boxes by Boolean operation between the extrusion of the monotone polygon and the box primitive.

In case of modeling a building with roofs, the CG module follows these steps: (1) Generate primitives of appropriate size, such as boxes, prisms or polyhedra that will form the various parts of the house. (2) Boolean operations applied to these primitives to form the shapes of parts of the house, for examples, making holes in a building body for doors and windows, a)

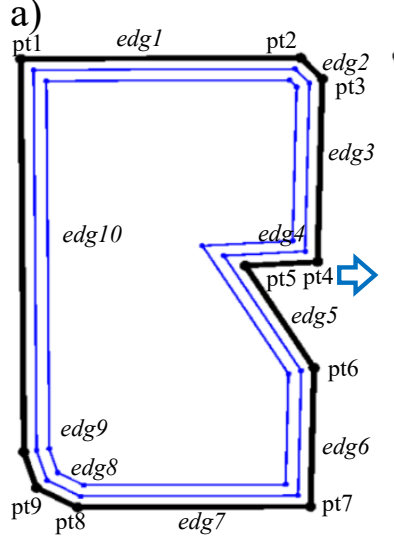

b)

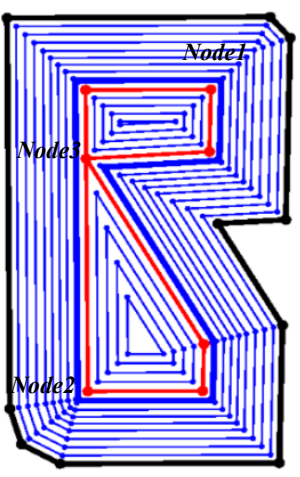

c)

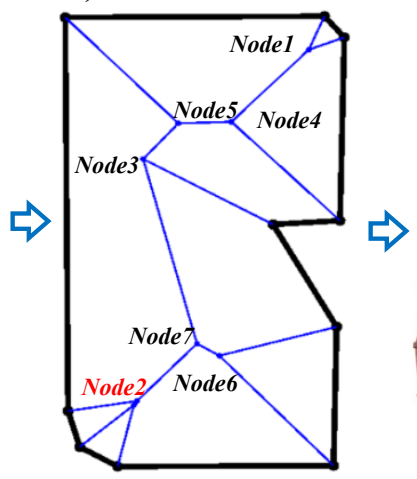

d)

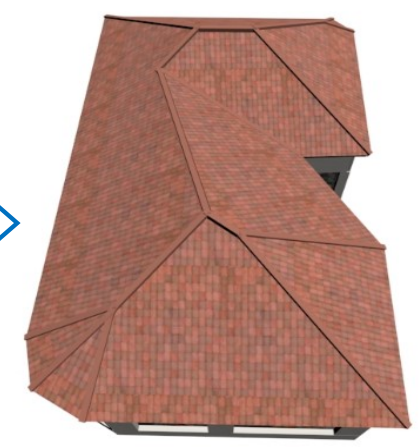

Figure 2: Shrinking process and a straight skeleton, a roof model generated. a) Input polygon (bold) start continuous shrinking process in which edges of the polygon move inward, parallel to themselves at a constant speed. b) Shrinking polygons (blue) by no event, and red one by a split event. c) The straight skeleton (blue) and monotone polygons. d) A roof model automatically generated: each roof board is based on an 'monotone polygon'. 
making trapezoidal roof boards for a hipped roof and a temple roof. (3) Rotate parts of the house according to the inclination of the partitioned rectangle. (4) Place parts of the house. (5) Texture mapping onto these parts according to the attribute received. (6) Copy the 2nd floor to form the $3 \mathrm{rd}$ floor or more in case of building higher than 3 stories.

CG module has been developed using Maxscript that controls 3D CG software (3ds MAX, Autodesk Inc).

\section{STRAIGHT SKELETON COMPUTATION}

Aichholzer et al. [Aic95] introduced the straight skeleton defined as the union of the pieces of angular bisectors traced out by polygon vertices during a continuous shrinking process in which edges of the polygon move inward, parallel to themselves at a constant speed. The straight skeleton is applied to constructing general shaped roofs based on any simple building polygon, regardless of their being rectilinear or not.

As shrinking process shown in Fig.2, each vertex of the polygon moves along the angular bisector of its incident edges. This situation continues until the boundary change topologically. According to Aichholzer et al. [Aic95], there are two possible types of changes:

(1) Edge event: An edge shrinks to zero, making its neighboring edges adjacent now.

(2) Split event: An edge is split, i.e., a reflex vertex runs into this edge, thus splitting the whole polygon. New adjacencies occur between the split edge and each of the two edges incident to the reflex vertex.

The shrinking procedure is uniquely determined by the distance $\mathbf{d}_{\text {shri }}$ between the two edges of before $\&$ after shrinking procedure.

The distance e $\mathbf{d}_{\text {shri }}$ is the $\mathbf{d}_{\text {shri }}$ when an Edge event happens in the shrinking process. e_d $\mathbf{d}_{\text {shri }}$ for the edge $\left(\mathrm{ed}_{\mathrm{i}}\right)$ is calculated as follows:

$\mathrm{e}_{-} \mathbf{d}_{\text {shri }}=\mathrm{L}_{\mathrm{i}} /\left(\cot \left(0.5 * \theta_{\mathrm{i}}\right)+\cot \left(0.5 * \theta_{\mathrm{i}+1}\right)\right)$

where $L_{i}$ is the length of ed $d_{i}$, and $\theta_{i} \& \theta_{i+1}$ are internal angles of vertices incident to $\mathrm{ed}_{\mathrm{i}}$.

When $0.5^{*} \theta_{i}+0.5^{*} \theta_{i+1}<180$ degrees, i.e. , the sum of the internal angles of two vertices incident to an edge is less than 360 degrees, an Edge event may happen unless the edge is intersected by an angular bisector from a reflex vertex and a Split event happens.

\subsection{How Straight Skeleton is formed}

How a straight skeleton and monotone polygons are formed is as follows.
(1) One simple polygon $(\mathbf{P})$ is given such as shown in Fig.2a. If there is any reflex vertex in the $\mathbf{P}$, then it can be divided into two or more polygons.

(2) The system calculates e_d $\mathbf{d}_{\text {shri }}$ (receding distance for an Edge event, shown in above (1)) for all edges and finds the shortest of them. Then, the system checks if a Split event occurs by increasing $\mathbf{d}_{\text {shri }}$ by (e_d $\mathbf{d}_{\text {shri }}$ $/ \mathrm{n}$ _step). In this way, the shrinking process may proceed until $\mathbf{d}_{\text {shri }}$ reaches the shortest e $\mathbf{d}_{\text {shri }}$ calculated.

(3) During shrinking until $\mathbf{d}_{\text {shri }}$ reaches the shortest

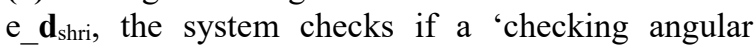
bisector' from a reflex vertex intersects another edge of the polygon or not. If an edge is found intersected, then the system calculates the node position by the Split event. The position of the node is calculated by the intersection of two angular bisectors: one from the reflex vertex and the other between the intersected edge and one of two edges incident to the reflex vertex. However, edges may be intersected by several 'checking angular bisectors' from several reflex vertices. Among the several reflex vertices, the reflex vertex that gives the shortest $\mathbf{d}_{\text {shri }}$ will be selected for calculating the node position.

(4) In the process of (2), a Split event may happen and the polygon will be divided into some polygons: Ps.

In this 'Split event checking' process, all divided polygons are checked if they can be divided more. As long as there are some Ps that can be divided, 'Split event checking' routine will continue. After that, the system concentrates on the Edge event procedure.

(5) In this stage, since the number of polygons divided does not increase by the Split event, the system can concentrate on the Edge event including Third and Line segment event procedures. If the polygon divided has only three vertices, then the polygon (triangle) collapses to a node; this is the final stage for the polygon divided.

(6) While the Edge events are being executed, the topology of the polygon will change. If the change happens, then the system re-implement the process from (2) to (5) for the polygon whose topology has changed. At that moment, the system recalculates the length of each edge and the internal angle of each vertex in order to find the shortest $\mathbf{d}_{\text {shri }}$ for next events. This re-implementation process continues until all polygons changed collapse to a node or a line segment.

\subsection{Node Structure}

The generated node will be associated with the edges of original $\mathbf{P}$ (original edge: o-edge) which are identified by original edges' ID (e.g. edgl \& edg2 in Fig.2a), since at least three original edges sweep to form a node. Therefore, at each event when the node is generated, at least three o-edges will be linked to the 
a)

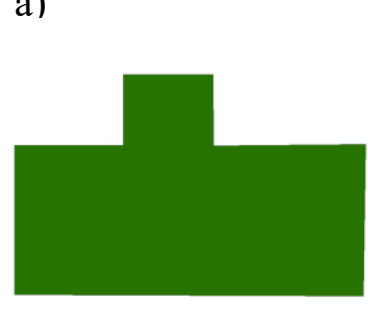

b)

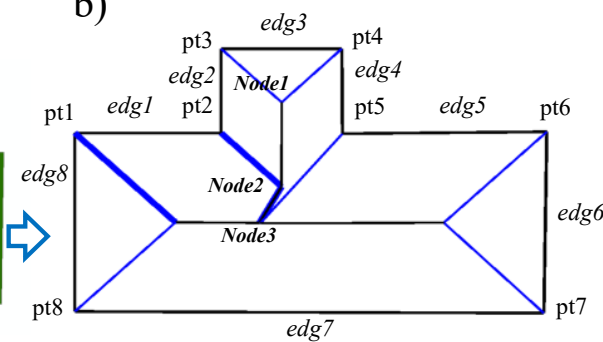

c)

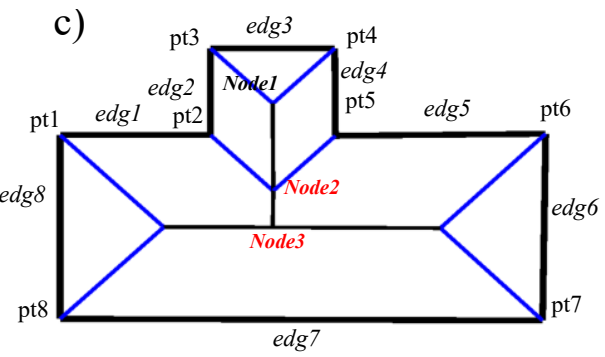

Figure 3: a) An orthogonal building polygon b) a monotone polygon with an acute angle c) rectified monotone polygons by 'Line segment' event

node. This means more than three o-edges ID will be stored in the node with a suitable structure.

In our system, a node has the following properties; (a) 'Node Type' (how the node is risen; by Edge event or Split event, Vertex event, Multiple Edge event and so on) (b) 'Number of forming edges' (usually three edges sweep to form a node, but more than three edges sweep in case of Multiple Edge event) (c) 'o-edge ID preceding the vanishing edge' (by Edge event) or 'oedge ID of one of the edge incident to the reflex vertex' (by Split event) (d) 'o-edge ID following the vanishing edge' (by Edge event) or 'o-edge ID of the other edge incident to the reflex vertex' (by Split event) (e) 'oedge ID of at least one vanishing edge' (by Edge event) or 'o-edge ID of a split edge' (by Split event)

Since three edges usually sweep into the node, three 'o-edge IDs' are stored in the property of a node. These IDs are used for forming a monotone polygon. The system is looking for the node which has the same 'o-edge ID' as each original edge of $\mathbf{P}$ to form monotone polygons.

In special cases, four or more edges collapse into nodes, such as Node2 in Fig. 2 and Node1,2,3 in Fig.4c. In extreme cases, such as a hexagon or a regular polygon, a star-shaped polygon collapses to a node, four or more o-edges will sweep into a node, and more than three 'o-edge IDs' are stored in the property of the node. Therefore, a node needs 'Number of forming edges' property.

This is the case of a multiple Edge event or the case Eppstein et al. [Epp99] defined as a 'degenerate case' in which the straight skeleton can have vertices of degree higher than three, introduced by simultaneous events at the same location. However, in single or double precision floating point calculation for the position of the node, it is quite rare for four or more vertices to reach the same point simultaneously.

To rectify monotone polygons to be appropriate shape for roof boards, in our system, if multiple edges collapse into a certain area considered as a point for a node, then they are considered to converge into the same point and the node is formed.

\subsection{Line Segment Event}

Since three edges usually sweep into a node, very often this causes a quite acute angle shape that is not appropriate for roof board shape shown in Fig.3. In Fig. $3 \mathrm{c}$, pt5 propagates to join pt 2 and four edges (edg1,2,4,5) propagate into Node2, whereas, in Fig.3b, pt5 does not join pt 2 and goes off Node2, and three edges $(e d g 1,4,7)$ result in Node3 with acute angle shape. This acute angle shape is also found at the
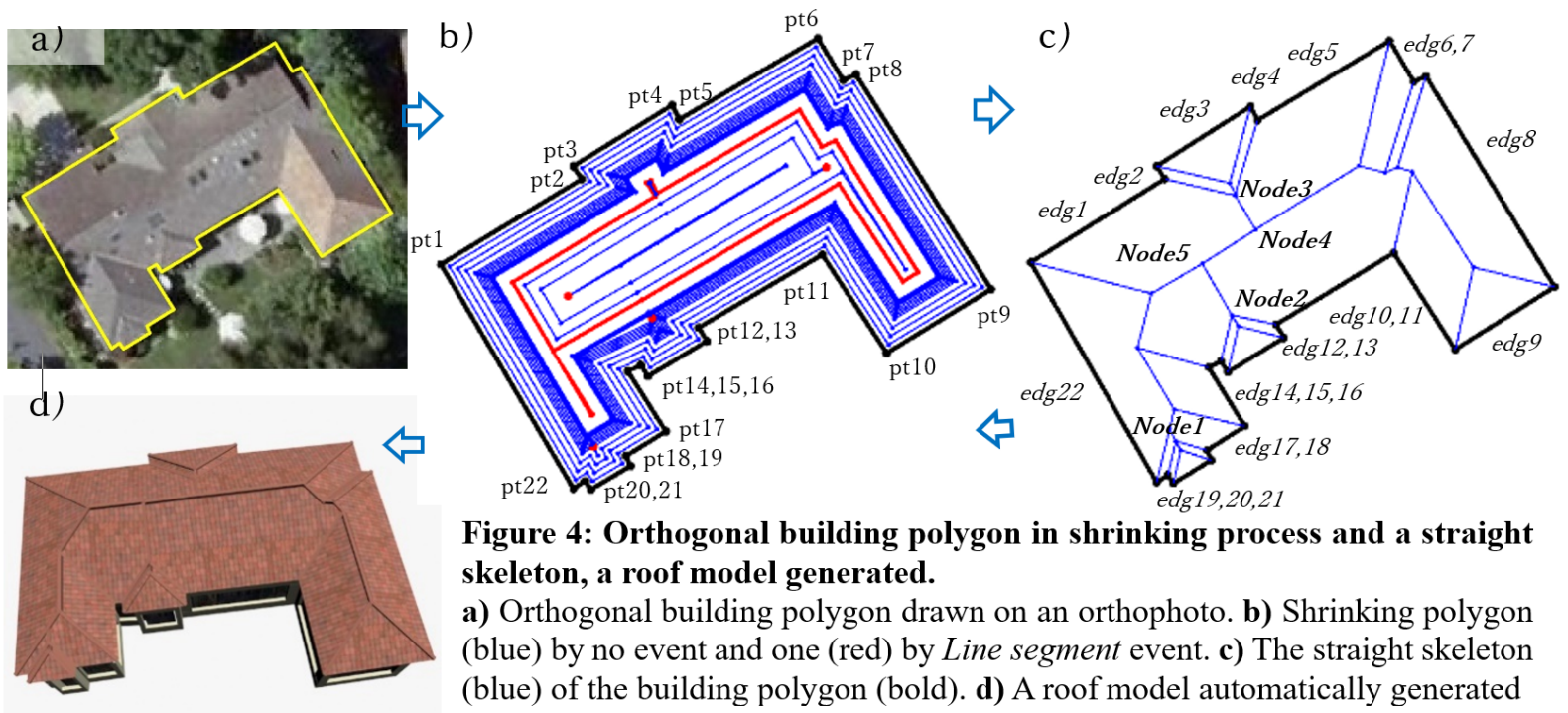

Figure 4: Orthogonal building polygon in shrinking process and a straight skeleton, a roof model generated.

a) Orthogonal building polygon drawn on an orthophoto. b) Shrinking polygon (blue) by no event and one (red) by Line segment event. c) The straight skeleton (blue) of the building polygon (bold). d) A roof model automatically generated 
figure of Eppstein et al. [Epp99], which uses perturbation techniques, replacing the high-degree node with several nodes of degree three, connected by zero-length edge. In our system, using the technique completely opposite to Eppstein's perturbation, a 'Line segment' event is proposed where edges are overlapped and collapse into a line segment instead of a node to avoid the acute angle shape. This so-called snapping function is done by setting up a certain range for possible 'Line segment' events, in which edges converge into a certain area considered as a line segment, then they are supposed to converge into the same line segment.

By a 'Line segment' event, two parallel edges converge into one edge (line segment), and the convergent line segment will be detached from a next shrinking body polygon. But if the detached line segment leaves no vertex for next shrinking process, then the line segment is disconnected from a body skeleton. Therefore, the detached line segment leaves at least one vertex for next shrinking process. Examples are shown in the line segment between Node2 and Node5 in Fig.4c and Fig.5b; one node whose interior angle is flat will remain for the next shrinking process so as to create the border of monotone polygons. For example, in Fig.4c \& Fig.5b, four edges $($ edg11,12,14,15) propagate into Node2, and two overlapping edges $(\operatorname{edg} 12,14)$ turn into the line segment incident to Node 2 \& nearby Node after edg13 disappeared.

If a configurable range is quite narrow, then edge propagation will be extended, ending in Node 5 as shown in Fig.5a; three edges $(\operatorname{edg} 12,14,15)$ result in Node2, and three edges $(e d g 11,12,15)$ result in Node 5 whose inner angle is quite acute, which is improper for roof board shape.

\subsection{Monotone Polygon Nodes Sorting}

According to Aichholzer et al. [Aic95], the area divided by a straight skeleton will be a "monotone polygon'. To get the monotone polygons, the set of the nodes belonging to each original edge will be sorted
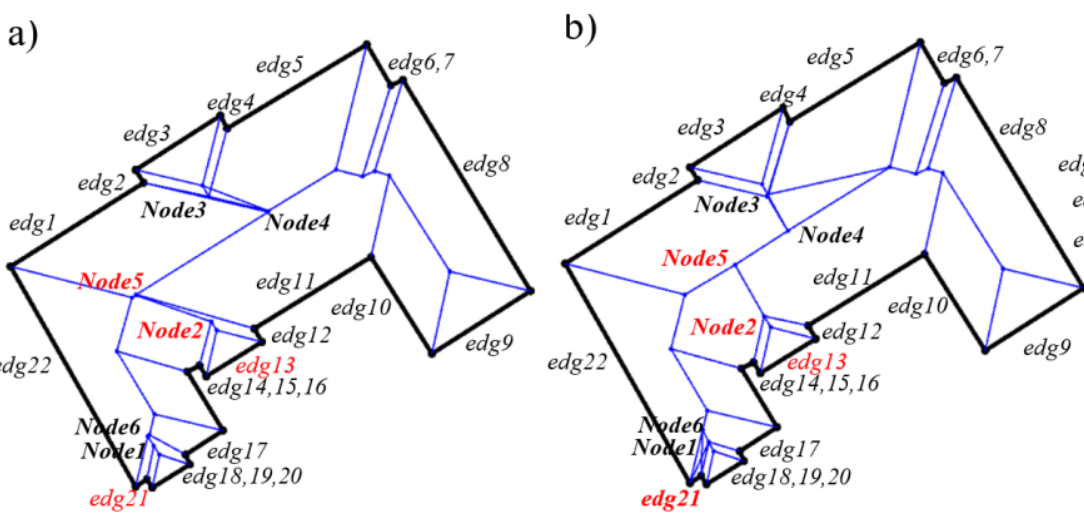

according to the 'coordinate value of node vector projections' onto the original edge vector parallel to each original edge. These nodes are coplanar and will form roof boards for a 3D building model. However, for some polygon, this methodology does not work, resulting in self-intersecting polygons. Fig.5c shows monotone polygons for edg13 are self-intersecting. This is because the edge (connecting Node $3 \&$ Node 4 ) of the monotone polygon is perpendicular to the original edge (edg13) of the polygon, and the nodes are connected in the order of 'node vector projection'. The self-intersection is found at edg29 in Fig.5c and edg 21 in Fig. 5 b.

To avoid self-intersection, the azimuth angle of the nodes belonging to the same monotone polygon is proposed, where the azimuth is the angle between each original edge vector and a node vector. The first node in the monotone polygon vertices numbering is selected from the node with least azimuth, and the last node is the node with greatest azimuth, since the nodes near the both ends on an original edge may wrap around both ends for some monotone polygons, and wrapping around nodes may not have simply increasing 'coordinate value'. For example, in Fig.5c, the edge (connecting Nodel \& Node2) of the monotone polygon is perpendicular to the original edge (edg29), and Nodel \& Node2 have the same 'coordinate value', resulting in self-intersection at nodes sorting. Thus, the nodes at ends are sorted by the azimuth angles. Then, the sorting of the nodes is found successful in a complicated shape polygon such as the one in Fig.4c and Fig.6c.

\section{APPLICATION}

Here are the examples of 3D building models automatically generated by the integrated system. Fig. 6 \& Fig. 7 show the examples of 3D building models automatically generated. In generating these models, we classify the case of 'Line segment event'. In Split event as mentioned in section 4.1, it is assumed to calculate the intersection of two non-parallel line

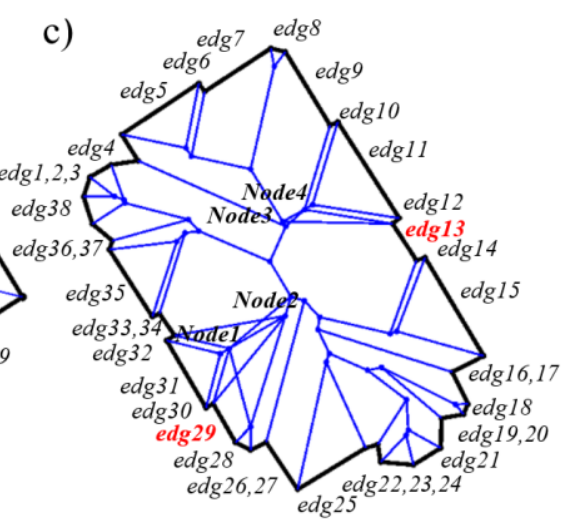

Figure 5: Monotone polygons with acute angle and self-intersecting monotone polygons. a) Some monotone polygons have acute angle if a Line segment event does not occur. b) \& c) Some monotone polygons are self-intersecting if the set of the nodes are sorted according to the 'node vector projections' onto the original edge vector. 
segments, i.e., two non-parallel angular bisectors. However, for some orthogonal polygons, two parallel edges will be overlapped when shrunk by e $\mathbf{d}_{\text {shri, }}$, and two parallel angular bisectors will be overlapped. If we do not classify the case of Line segment event, then we end up with numerical error by trying to calculate the intersection of two parallel line segments.

Once 3D models with roofs are created, a top view of these models can be a roof report as shown in Fig.7i \& $7 \mathrm{j}$, which can be used for the rapid assessment of roof damages by insurance companies. Automated generation of simple and complex roof geometries will be utilized for rapid roof area damage reporting by the length measurements and area calculations of all roof surfaces. The roof board area will be easily calculated, since a roof board is a monotone polygon, and can be partitioned into a set of trapezoids or triangles. The roof board area will be calculated by adding these trapezoids, and subtracted or added by two triangles, depending on the shape of the monotone polygon.

The advantage of our generation system is that our $3 \mathrm{D}$ building models created are utilized for architectural design, i.e., BIM (Building Information Model), while 3D models created by procedural modeling are not solid models but surface models which are to be converted into geometric primitives (CSG) when they are used for construction design.

Now, architectural design world is experiencing a shift from 2D CAD drawings to BIM 3D modeling. BIM revolution is happening in the construction industry that is producing a step change in efficiency and accuracy. In our research, 3D building models automatically created can be used for BIM 3D modeling. There is no automatic generation system for 3D building solid models with complicated roofs as far as we know. Automatic generation will be compared with manual creation which are a series of manual operations mentioned in section 1 by $3 \mathrm{ds}$ Max, and broken down into functions of the program (Maxscript) described as CG module's process in section 3. It will take about more than one hour to create one hipped roof house including making intricately shaped ridges, while several seconds to automatically generate one by the personal computer. If given digital maps with attributes being inputted, as shown in Fig. 1 \& 7 , the system automatically generates one hundred 3D building models within less than 10 minutes by the personal computer with Intel(R) Core(TM) i7-7820HK CPU $2.90 \mathrm{GHz}$.

\section{CONCLUSION}

In this paper, the new and extended methodology is proposed for adding 'Line segment' event besides the conventional events, and 'monotone polygon nodes sorting' by which self-intersecting monotone polygons are not formed. Thus, the proposed integrated system succeeds in automatically generating 3D building models.

The roofs created by the straight skeleton are limited to hipped roofs with their roof ridges parallel to nearby long edges of the building contour. However, there are many roofs whose ridges are perpendicular to long edges. In the residential area all over the world, there are many roofs the straight skeleton method cannot create. For example, in the middle of the top edge in Fig.4a and Fig.6a, Fig.7e, there are some branch roofs which are not slanting and drop 90 degrees vertically, i.e., gable roofs. These are not created by the straight skeleton.

In order to create various shape of roof, we propose a couple of schemes to create roofs by straight skeleton computation or partitioning or separating of orthogonal polygons. A complicated orthogonal building polygon can be partitioned or separated into a set of rectangles. Our proposed system partitions orthogonal polygons into a set of rectangles and places various shapes of roofs which include gable $\&$ hipped roofs or the roof whose ridges are perpendicular to nearby long edges on these partitioned rectangles. Thus, in order to create the 3D building models that have hipped and gable branch roofs, the future work is for developing the system in which we can select a couple of various schemes; dividing or separation

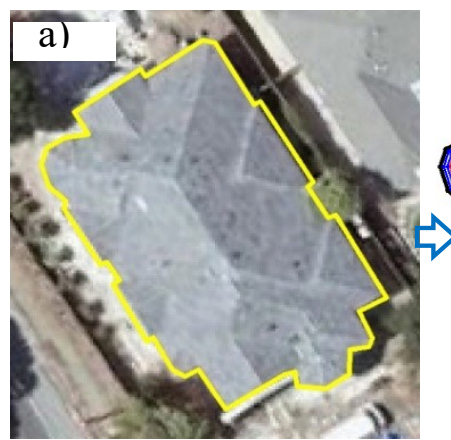

b)

b)

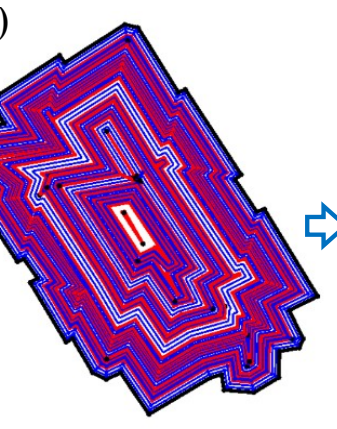

c)

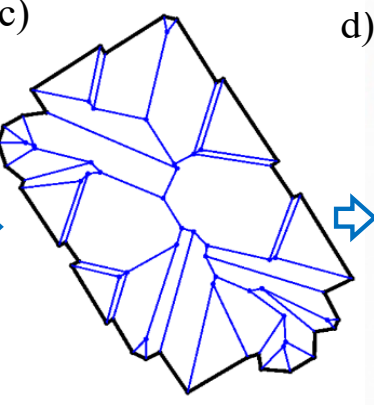

d)

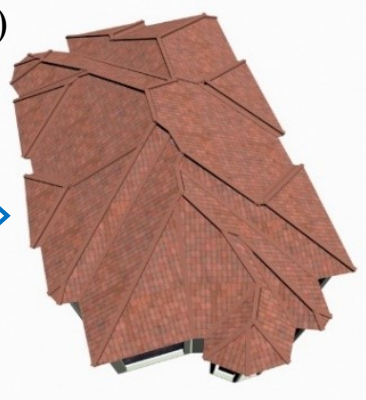

Figure 6: Complicated shape polygon in shrinking process and a straight skeleton, a roof model generated.

a) Complicated building polygon drawn on an orthophoto. b) Shrinking polygons (blue) by no event and red ones by events. c) The straight skeleton (blue) of the building polygon (bold). d) A roof model automatically generated 
scheme or straight skeleton scheme to automatically create the different styles of roofs for one building footprint.

\section{REFERENCES}

[Aic95] Aichholzer O., Aurenhammer F., Alberts D., Gärtner B. A novel type of skeleton for polygons. Journal of Universal Computer Science (1995), 1 (12): 752-761.

[Ede14] Johannes Edelsbrunner, Ulrich Krispel, Sven Havemann, Alexei Sourin. Constructive Roof Geometry. Cyberworlds (CW) 2014, DOI: 10.1109/CW.2014.17

[Epp99] Eppstein David, Erickson Jeff. Raising roofs, crashing cycles, and playing pool: applications of a data structure for finding pairwise interactions. Discrete and Computational Geometry (1999), 22 (4): 569-592

[Hel17] Helda M., Palfradera P. Straight Skeletons with Additive and Multiplicative Weights and Their Application to the Algorithmic Generation of Roofs and Terrains. Computer-Aided Design, Elsevier B.V. (Nov 2017), Vol 92, pp. 33-41

[Jia09] Jiang N., Tan P., Cheong L. F. Symmetric architecture modeling with a single image. Proc. SIGGRAPH Asia '09 (2009), papers No. 113

[Kel11] Kelly T., Wonka P. Interactive Architectural Modeling with Procedural Extrusions. ACM Transactions on Graphics (TOG) (2011), 30(2), 14-28.

[Mue06] Mueller P., Wonka P., Haegler S., Ulmer A., Gool L.V. Procedural modeling of buildings. ACM Transactions on Graphics (2006), 25, 3, 614-623.

[Par01] Parish Y. I. H., Müller P. Procedural modeling of cities. Proceedings of ACM SIGGRAPH (2001), ACM Press, E. Fiume, Ed., New York, 301-308 


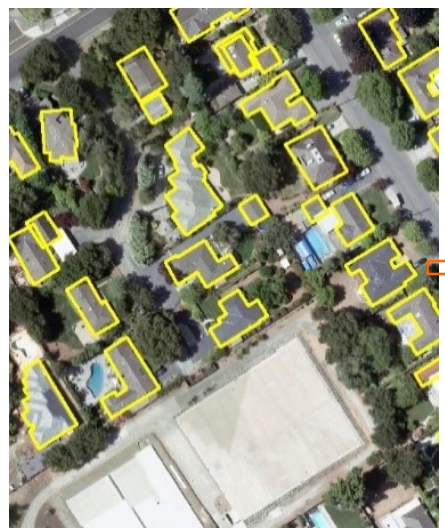

(a) Building polygons on 2D Digital Map: Most of them are orthogonal.

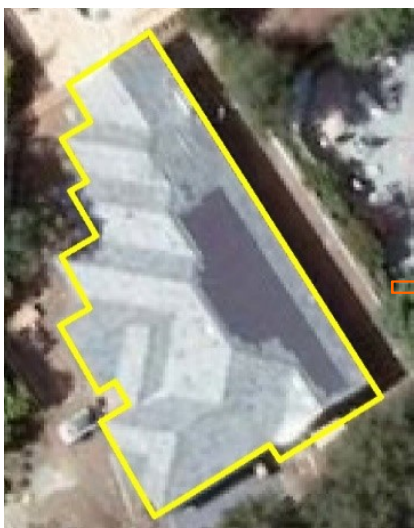

(e) Enlarged building polygons: orthogonal

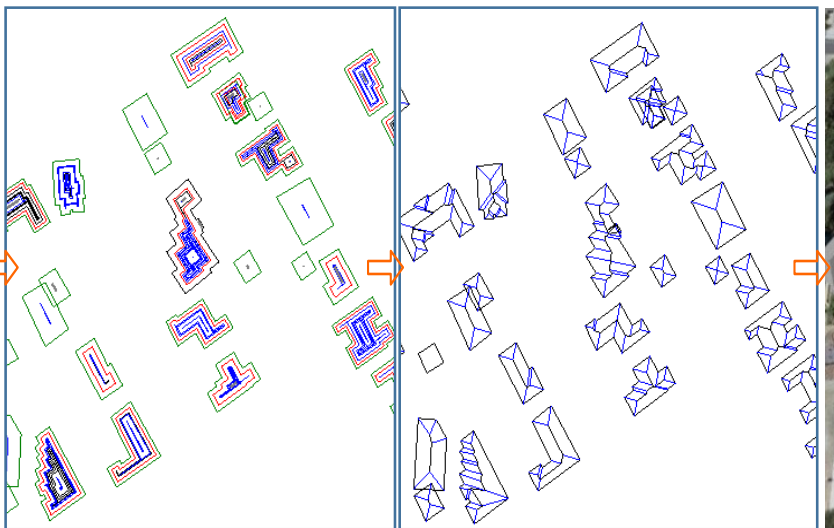

(b) Set of receding (c) The straight skeleton $\begin{array}{ll}\text { polygons by } \mathbf{d}_{\text {shri }} \text { calculated formed as the union of the } \\ \text { by equation (1) } & \text { pieces of angular bisectors }\end{array}$ (d) Automatically generated

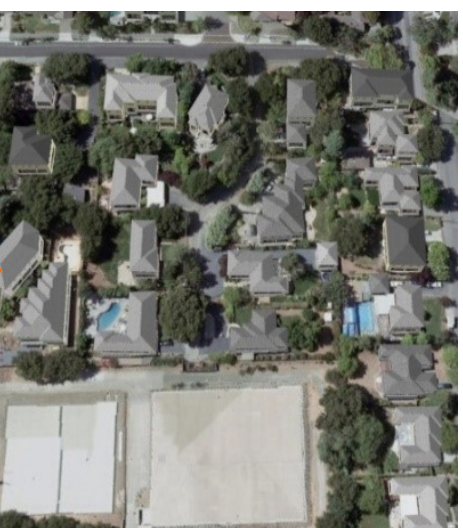

3D building model based on monotone polygons

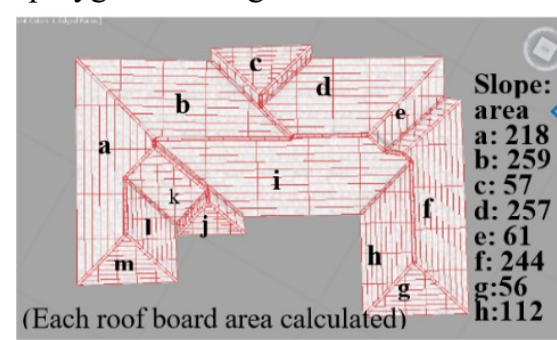

(i) Automatically created roof report for damage evaluation
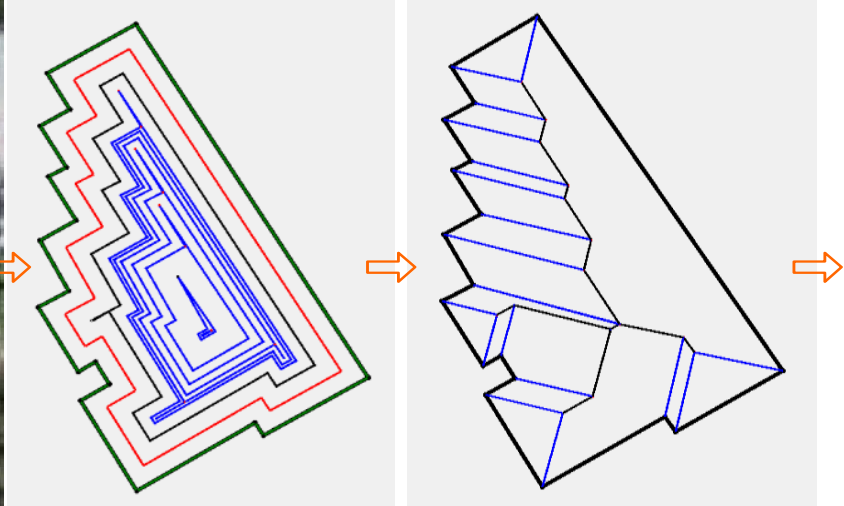

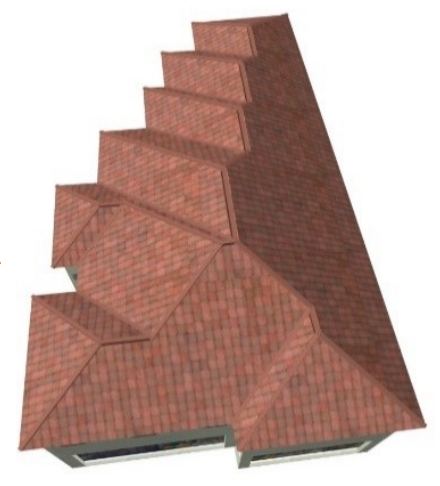
pieces of angular bisectors monotone polygons (f) Set of receding polygons by (g) The straight skeleton (h) Automatically generated
$\mathbf{d}_{\text {shri }}$ calculated by equation (1) formed as the union of the 3D building model based on ated by equation $(1)$
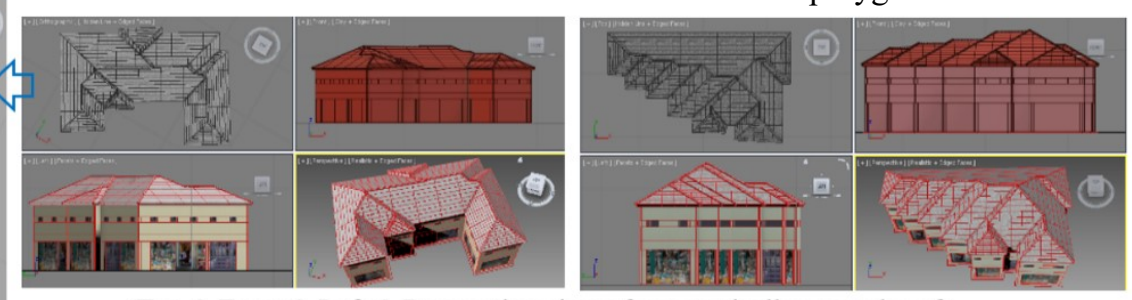

(Top \& Front \& Left \& Perspective view of automatically created roof)

(j) Ground [floor] plan (Top view) of automatically created 3D building model

Figure 7: Shrinking process and a straight skeleton, a roof model generated, roof report 